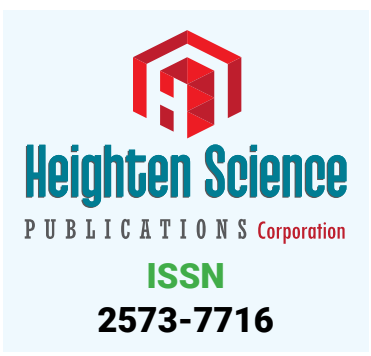

*Address for Correspondence: Dr. Diego Abelardo Alvarez Hernández, Faculty of Health Sciences, Universidad Anahuac México Norte, Av. Universidad Anahuac 46, Col. Lomas Anahuac, Naucalpan de Juarez, Estado de México, México, Email:

diego.alvarez.hernandez@hotmail.com

Submitted: 19 July 2017

Approved: 26 July 2017

Published: 27 July 2017

Copyright: @ 2017 Hernandez DAA, et al. This is an open access article distributed under the Creative Commons Attribution License, which permits unrestricted use, distribution, and reproduction in any medium, provided the original work is properly cited

Keywords: Gangosa; Gondou; Treponema pallidum; Pian; Yaws
Editorial

\section{Yaws essentials: What health professionals should know about yaws}

\author{
Diego-Abelardo Alvarez-Hernandez ${ }^{1-3 *}$ and Alexia S Rivera ${ }^{1,2}$ \\ ${ }^{1}$ Faculty of Health Sciences, Universidad Anahuac Mexico Norte, Mexico State, Mexico \\ ${ }^{2}$ Research \& Academic Coordination, Medical Impact, Mexico State, Mexico \\ ${ }^{3}$ Coordination of Medical Services, Mexican Red Cross PAR Huixquilucan Office, Mexico State, \\ Mexico
}

Yaws is recognized by the World Health Organization (WHO) as 1 of the 20 Neglected Tropical Diseases (NTDs), a group of communicable diseases that have subsisted in tropical and subtropical environments, and that affect people living in poor and marginalized societies [1]. Yaws also form part of a group of chronic bacterial infections, commonly known as the endemic trepanomatoses. These diseases are caused by a spiral bacteria of the genus Treponema, which also includes bejel and pinta, being yaws the most common [2]. Like syphilis, yaws have been described in three stages; primary stage characterized by granulomatous skin lesions, secondary stage by generalized spread, and tertiary stage by chronic destructive disease of skin, cartilages and bones [3].

Yaws is the second trepanomatoses in prevalence, after the one caused by $T$. pallidum pallidum (syphilis), and before the ones caused by T. pallidum subespecies endemicum (bejel) and T. carateum (pinta) [2,4]. In 2015, a total of 65,000 cases were reported through 13 countries, [5] with $85 \%$ of them happening in Ghana, Papua New Guinea, and Solomon Islands [6]. However, the exact prevalence and burden of the disease remains unclear due to various factors, including low completeness/timeliness of reporting, the inconsistent reporting of cases across countries because of the status of non-notifiable disease, misdiagnosis, and lack of accuracy of data [7].

Yaws is a bacterial disease caused by T. pallidum subespecies pertenue. T. pallidum is a gramnegative bacteria which divides slowly, cannot be cultured in vitro, and cannot survive outside the mammalian host [8]. It favors from warm, humid and moist climates, flourishing at best in countries with a mean annual isotherm of $27^{\circ} \mathrm{C}$ and over [9]. Although its closely related to syphilis, it is not spread by sexual contact, but by skin-to-skin contact through abrasions or cuts with infectious lesions [7]. Humans are generally considered the sole reservoir, although a few reports exist of the presence of the disease among non-human primates [10].

Living between the tropics, in rural and poor communities, low standards of hygiene, heavy rainfall, and trauma in lower extremities, are considered risk factors for acquiring the disease. Furthermore, children between 2 and 15 years old seem to be the most affected [7].

The disease is usually acquired during childhood, mostly before puberty, with adult disease being a manifestation of earlier infection. The clinical manifestations of yaws can be classified into primary, secondary and tertiary stages [9]. The primary stage has an incubation period of 9 to 90 days [11]. During the primary stage, a lesion called "mother yaw" develops as a papilloma at the inoculation site, which after 14 to 28 days 
enlarges and ulcerates [4]. The most common sites for this "mother yaw" are ankles and legs, but it may be found on the face, arms, hands and buttocks [7]. Resolution and lesion healing without treatment occurs after almost 6 months. Regional lymphadenopathy and arthralgia may occur; [4] The secondary stage develops after 1 to 24 months from disease onset [8]. During the secondary stage, multiple lesions called "daughter yaws" develop as smaller skin papillomas, which are widespread [12]. Hyperkeratosis, cracking and secondary infections may also develop at the soles [7]. Fever, malaise, measles-like eruption, papillomas in moist areas, and osteoperiostitis involving long bones and fingers may occur; [13] As in all treponemal infections, untreated patients may develop latent infection, with positive serology but no clinical signs; [8] Finally, in $10 \%$ of the cases, the tertiary stage may develop after 5 to 10 years from disease onset, [14] causing destruction of tissue, cartilage and bones. Subcutaneous gummatous nodules, exostoses of the maxillary bones (goundou), destructive osteitis of the septum and palate (gangosa), monodactylitis, juxta-articular nodules, and apparent bowing of the tibia (saber shin) may be develop, leading to disfigurement and disability $[4,8]$.

Complications that otorhinolaryngologists could help manage are gangosa and gondou. Gangosa or rhinopharyngitis mutilans, is an uncommon form of tertiary yaws which presents as nasal or palatal ulceration. It is a destructive ulcerative rhinopharyngitis that usually begins at the mucocutaneuos junction of the nasal septum with an inwardly spreading ulceration to destroy the nose, palate and pharynx, sparing the premaxilla, upper lip and larynx. On the other hand, Gondou is a more uncommon form of tertiary yaws which presents as bilateral oval swellings in the nasal processes of the maxilla. It is a subperiosteal deposition of new bone [15]. Both conditions cause cosmetic deformities that may require surgical correction. In some cases, the maxillary bony swellings may be locally removed and the external nasal deformity may be improved by augmentation rhinoplasty, assuming adequate initial chemotherapeutic treatment [3].

Yaws diagnosis is based in the combination of emergence of the disease in an endemic area, clinically typical papilloma lesions, and paraclinicals with seroreactivity in a treponemal test [16]. On the field, epidemiological background and clinical manifestations should be considered. However, if laboratory tests are available, direct detection (dark-field microscopy or rabbit infectivity test), molecular (polymerase chain reaction), and serological non-treponemal (rapid plasma regain and venereal disease research laboratory) and treponemal (indirect immunofluorescence, western blot, automated enzyme, chemiluminiscent or multiplex flow immunoassays) methods serve to confirm the diagnosis. Furthermore, several point-of-care tests have become available, and require minimal equipment and training. Differential diagnosis of primary and secondary stages should be done with other causes of skin diseases like chromomycosis, cutaneous leishmaniasis, ecthyma, impetigo, leprosy, molluscum contagiosum, planar warts, psoriasis, sarcoidosis, scabies, tropical ulcers and tungiasis, meanwhile for the tertiary stage, it should be done with rhinopharyngeal lesions like mucocutaneous leishmaniasis, leprosy, rhinoscleroma, rhinosporidiosis and tuberculosis, and with bone lesions like the ones caused by African histoplasmosis, bacterial osteomyelitis, bejel, sickle cell anemia, syphilis and tuberculosis [4].

Azithromycin is the first-line treatment against T. pallidum subespecies pertenue infection, but benzathine penicillin can be administered for patients allergic to azithromycin or for patients where treatment with azithromycin has failed. The dosage regimen for azithromycin is $30 \mathrm{mg} / \mathrm{kg}$ body weight per month in a single dose, meanwhile for benzathine penicillin is 600,000 units for children aged under 10 years and 1,200,000 units for people aged over 10 years in an intramuscular injection in a single dose [2]. Based on the level of seropositivity among children, targets for treatment of the community in low endemic countries include active cases and their contacts in households, schools and workplaces, meanwhile in moderate endemic 
countries include active cases, their contacts and all children below 15 years old [14]. Follow-up is recommended for at least 4 months after completing antibiotic treatment to confirm cure, assess possible complications, and observe any recurrences. In over $95 \%$ of cases, complete healing will be observed [2]. However, no long-term immunization can be acquired upon treatment, and therefore, reinfection is possible [17]. Furthermore, there is not any prophylactic or therapeutic vaccine available for yaws [2].

Yaws is an infectious, debilitating and disfiguring disease of poverty that mainly affects children in rural communities in tropical areas [18]. It causes a chronic relapsing nonvenereal treponematoses, characterized by highly contagious primary and secondary cutaneous lesions, and noncontagious tertiary destructive lesions of the bones [19]. Mass campaigns carried out in yaws endemic areas since the 1950's gave arise to the possibility of eradication, however, resurgence of yaws has been reported due to curtailment of control activities, which has allowed the reservoir of untreated cases to grow unchecked [12]. Yaws presents new challenges such as unknown epidemiological situation, poor awareness and knowledge among healthcare workers, and attenuated clinical forms of the disease [10]. General practitioners and specialists dedicated to diagnose and treat patients between the tropics, especially in endemic countries, should be aware of yaws and its complications. Currently, there is not enough nor recent information about the development of tertiary stage complications, and which surgical techniques should be used to treat them. Otorhinolaryngologists and maxillofacial surgeons can really make a difference for patients who suffer from them, being both able to improve their life quality, and to reduce the stigma caused by the disease.

\section{REFERENCES}

1. Neglected Tropical Diseases. WHO. 2017. Ref.: https://goo.gl/jevbSR

2. Yaws. WHO. 2017. Ref.: https://goo.gl/W9agsx

3. Whittet HB, Quiney RE. Nasal manifestations of yaws. J Laryngol Otol. 1988; 102: 1147-1149. Ref.: https://goo.gl/6fx $1 \mathrm{Xj}$

4. Giacani L, Lukehart SA. The Endemic Treponematoses. Clin Microbiol Rev. 2014; 27: 89-115. Ref.: https://goo.gl/pcjt8e

5. Mitjà $\mathrm{O}$, Houinei $\mathrm{W}$, Moses $\mathrm{P}$, Kapa $\mathrm{A}, \mathrm{Paru} \mathrm{R}$, et al. Mass treatment with single-dose azithromycin for yaws. N Engl J Med. 2015; 372: 703-710. Ref.: https://goo.gl/fRdqTa

6. Global health observatory data repository: Yaws. WHO. 2014. Ref.: https://goo.gl/Xp1hST

7. Kazadi WM, Asiedu KB, Agana N, Mijtà O. Epidemiology of yaws: an update. Clin Epidemiol. 2014; 6: 119-128. Ref.: https://goo.gl/6xop9e

8. Marks M. Yaws: towards the WHO eradication target. Trans R Soc Trop Med Hyg. 2016; 110: 319320. Ref.: https://goo.gl/KEq8Gp

9. Hill KR. Non-specific factors in the epidemiology of yaws. Bull World Health Organ. 1953; 8: 17-51. Ref.: https://goo.gl/NopJYa

10. Capuano C, Ozaki M. Yaws in the Western pacific region: a review of the literature. J Trop Med. 2011; 1: 1-15. Ref.: https://goo.gl/cd55m5

11. Perine PL, Hopkins DR, Niemel PLA, St John R, Causse G, et al. Handbook of endemic treponematoses: yaws, endemic syphilis, and pinta. WHO. 1984. Ref.: https://goo.gl/dRCwDW

12. Manirakiza A, Vilas-Boas S, Beyam N, Zadanga G, Konamna FX, et al. Clinical outcome of skin yaws lesions after treatment with benzathine benzylpenicillin in a pygmy population in Lobaye, Central African Republic. BMC Research Notes. 2011; 4: 543. Ref.: https://goo.gl/5W8evR

13. Mitjà $\mathrm{O}$, Hays $\mathrm{R}$, Ipai $\mathrm{A}$, Wau $\mathrm{B}$, Bassat $\mathrm{Q}$. Osteoperiostitis in early yaws: case series and literature review. Clin Infect Dis. 2011; 52: 771-774. Ref.: https://goo.gl/LEWzXP

14. Amin R, Sattar A, Basher A, Faiz MA. Eradication of yaws. J Clin Med Res. 2010; 2: 49-54. Ref.: https://goo.gl/4D7gzE 
15. Trenouth MJ. Goundou-tertiary yaws in the maxilla. British Journal of Oral Surgery. 1975; 113: 166171. Ref.: https://goo.gl/FipV5M

16. Martin PM, Gonzalez JP, Martin MH, Georges-Courbot MC, Palisson MJ, et al. Clinical aspects and usefulness of indirect absorbed immunofluorescence for diagnosis of yaws in Central Africa. J Clin Microbiol. 1988; 26 : 2432-2433. Ref.: https://goo.gl/QEZJxu

17. Rinaldi A. Yaws Eradication: Facing Old Problems, Raising New Hopes. PLoS Negl Trop Dis. 2012; 6: 1-4. Ref.: https://goo.gl/Qpw6Jx

18. Boock AU, Awah PK, Mou F, Nichter M. Yaws resurgence in Bankim, Cameroon: The relative effectiveness of different means of detection in rural communities. PLoS Negl Trop Dis. 2017; 8: 1-14. Ref.: https://goo.gl/kDJePp

19. Mitjà O, Asiedu K, Mabey D. Yaws. Lancet. 2013; 381: 763-773. Ref.: https://goo.gl/72ttiX 